\title{
Research on Innovative Design of Product Packaging Based on Big Data Technology
}

\author{
Fang Gan $\mathbb{D}^{1,2}$ Nurul Hanim Romainoor, ${ }^{1}$ and ZhiMin Guo ${ }^{3}$ \\ ${ }^{1}$ School of the Arts, Universiti Sains Malaysia, Penang 11800, Malaysia \\ ${ }^{2}$ School of the Arts, Guangxi Normal University for Nationalities, Chongzuo, Guangxi 532200, China \\ ${ }^{3}$ Nortel Instrument Co., Ltd, Shenzhen 515100, China \\ Correspondence should be addressed to Fang Gan; ganfang@gxnun.edu.cn
}

Received 24 November 2021; Revised 3 December 2021; Accepted 13 December 2021; Published 17 February 2022

Academic Editor: Baiyuan Ding

Copyright (C 2022 Fang Gan et al. This is an open access article distributed under the Creative Commons Attribution License, which permits unrestricted use, distribution, and reproduction in any medium, provided the original work is properly cited.

Traditional product packaging design mainly relies on the designer's personal experience and intuition, but there are problems such as uncontrollable content and lack of knowledge and guidance in the field of product design. With the development of big data technology, product packaging design in the era of big data is carried out under the support of a large amount of real data, which has high predictability, success rate, and short development cycle. Compared with structured data, unstructured data such as text, images, and audio has a higher value. Among them, text big data and image big data have good application prospects in the field of product packaging design due to their easy data acquisition, mature processing technology, and simple operation. This paper proposes a combination of big data technology and neural style transfer model and proposes an innovative design method for product packaging that can generate high-quality images and controllable content. First, the perceptual engineering theory is used to obtain user needs, to build a mapping model between product modeling elements and product semantics, and to guide the selection of product semantics and style maps; second, use neural style transfer models to reconstruct and combine the color features of style maps. After the integration, it migrated to product packaging design, based on big data product packaging innovation design methods, and developed a product innovation design auxiliary prototype system based on actual needs to improve the company's R\&D and innovation capabilities, shorten product development cycles, and reduce R\&D costs. Improve product success rate and user satisfaction.

\section{Introduction}

With the advent of the 21st century, the design has truly been integrated into all aspects of our lives. People are carrying out design activities all the time to obtain a more comfortable living environment. Standardized and mechanized production, on the one hand, enables the market citizens to purchase aesthetic products with lower expenditures and increases the quality of life through the quantification of products; on the other hand, due to the impact of economic globalization, the design is consistent. The international style lacks a clear personality. The design of "form follows function" and "equal emphasis on form and function" brings people a wealth of material enjoyment, but it neither bring people's spiritual warmth and considerate comfort nor can it satisfy consumers' demand for individualization and uniqueness. Because of demand, more and more people are buying "works" instead of "commodities." [1-4]. In the context of modern design, the design not only satisfies functions and people's surface visual experience but also needs to convey a certain emotional experience to consumers through design language.

Traditional product appearance innovation design mainly relies on the designer's personal experience and intuition and has defects such as strong subjectivity, weak interpretability, low predictability, long development cycle, slow response to user needs, and low product success rate. The advent of the era of big data has made it possible to improve product design capabilities. Big data contain a lot of valuable information such as product feedback, user preferences, market demand, and visual display. This information plays an important role in guiding product 
appearance innovation and design. Use the valuable information in big data to quickly, accurately, and fully capture. Responding to user needs has become the focus and hotspot of domestic and foreign research. This article tries to use big data technology to realize the design of personalized packaging system.

\section{Related Work}

With the rapid development of the Internet, the Internet of Things, and communication technologies, the amount of global data has exploded, and mankind has officially entered the era of big data. Big data technology describes a new generation of technology and architecture system, through high-speed collection, discovery, or analysis, to extract the economic value of various large amounts of data. The Gartner organization summarized big data as a massive, high growth rate, and diversified information asset that requires new processing models to enhance decision-making, insight, and process optimization capabilities. Academician Xu Zongben defined big data as "incapable of centralized storage, and it is difficult to analyze and process within an acceptable time, in which individual or part of the data presents a low value, while the data as a whole present a massive and complex dataset with a high value." Although the above definitions have different perspectives and focuses, they all reflect that big data are a dataset with the characteristics of large data volume, multiple data types, high data authenticity, fast data generation speed, high value, and low value [5-10].

At present, with the development of artificial intelligence technologies such as machine learning, deep learning, natural language processing, and computer vision, big data have been widely used, such as transportation, health care, fault diagnosis, tourism, and product design, and achieved certain results. Achievement: in the product design, traditional product design mainly relies on the designer's personal experience and intuition. Product design in the era of big data is carried out under the support of a large amount of real data, which has high predictability, success rate, and short development cycle. Compared with structured data, unstructured data such as text, images, and audio have a higher value. Among them, text big data and image big data have good application prospects in the field of product design due to their easy data acquisition, mature processing technology, and simple operation. Specifically, in terms of data research, text big data can make up for the limitations of traditional research methods in the number of surveys, geographical scope, time interval, and timeliness of data, ensuring the authenticity, reliability, and timeliness of survey information; in products, in terms of design plan visualization, image big data can directly generate product images, providing users and designers with intuitive visual displays. The current product design is developing in the direction of intelligence, systematization, and short development cycle. Making full use of the value contained in big data and introducing cutting-edge technologies such as machine learning and deep learning are the hotspots and difficulties of current product innovation design.

\section{Related Theoretical Methods}

3.1. Big Data Technology. With the development of social economy, the levels of user demand for products will become more diversified and individualized. Researchers must dare to break through the shackles of inherent concepts and break conventions to find new ideas and new ways of the product design. Big data contain a lot of valuable information, which is of great significance to product innovation and design. Figure 1 shows the life cycle data of the entire big data product [11-14].

3.2. Neural Style Transfer. Batch normalization (BN), IN, $\mathrm{CIN}$, and AdaIN are commonly used methods in neural style transfer. BN calculates the characteristic data (mean and variance) of each channel of a batch of samples, while IN calculates the characteristic data of each channel and sample independently. CIN performs style transfer by panning and zooming the normalized result. Each style needs to learn two parameters that control panning and zooming. AdaIN is obtained by improving CIN, and AdaIN calculates the control parameters adaptively from the feature representation of the image. Figure 2 shows the neural style transfer structure, using the "encoder-AdaIN-decoder" architecture, which mainly includes three parts: encoder, AdaIN, and decoder [15-17].

The encoder part adopts the VGG-19 network pretrained on the ImageNet dataset, which can detect the features in the image. The AdaIN layer normalizes the content map and matches the mean and variance of each channel feature map in the style map by aligning the mean and variance of each channel feature map in the content map:

$$
\begin{aligned}
t & =\operatorname{AdaIN}(f c, f s) \\
& =\sigma(f s)\left[\frac{f c-\mu f c}{\sigma(f(c))}\right]+\mu(f(s)),
\end{aligned}
$$

where $f$ represents the encoder, $c$ and $s$ represent the content map and the style map, respectively, and $\mu$ and $\sigma$ represent the mean and variance, respectively. The decoder part converts the feature space to the image space to generate a stylized image $T(c, s)$ :

$$
T(c, s)=\mathrm{g}(t)
$$

where grepresents the decoder.

The decoder adopts a network structure symmetrical to the encoder, and what needs to be trained in the entire network is the weight parameter of the decoder network. The loss function of the network is mainly composed of content loss and style loss. The training goal is to minimize the loss function [18-20]:

$$
L=L_{c}+L_{s},
$$

where $L$ is the loss function and $L_{c}$ and $L_{s}$ are content loss and style loss, respectively. The content loss is the Euclidean distance $\left(L_{2}\right.$ norm $)$ between the target feature and the input image feature: 


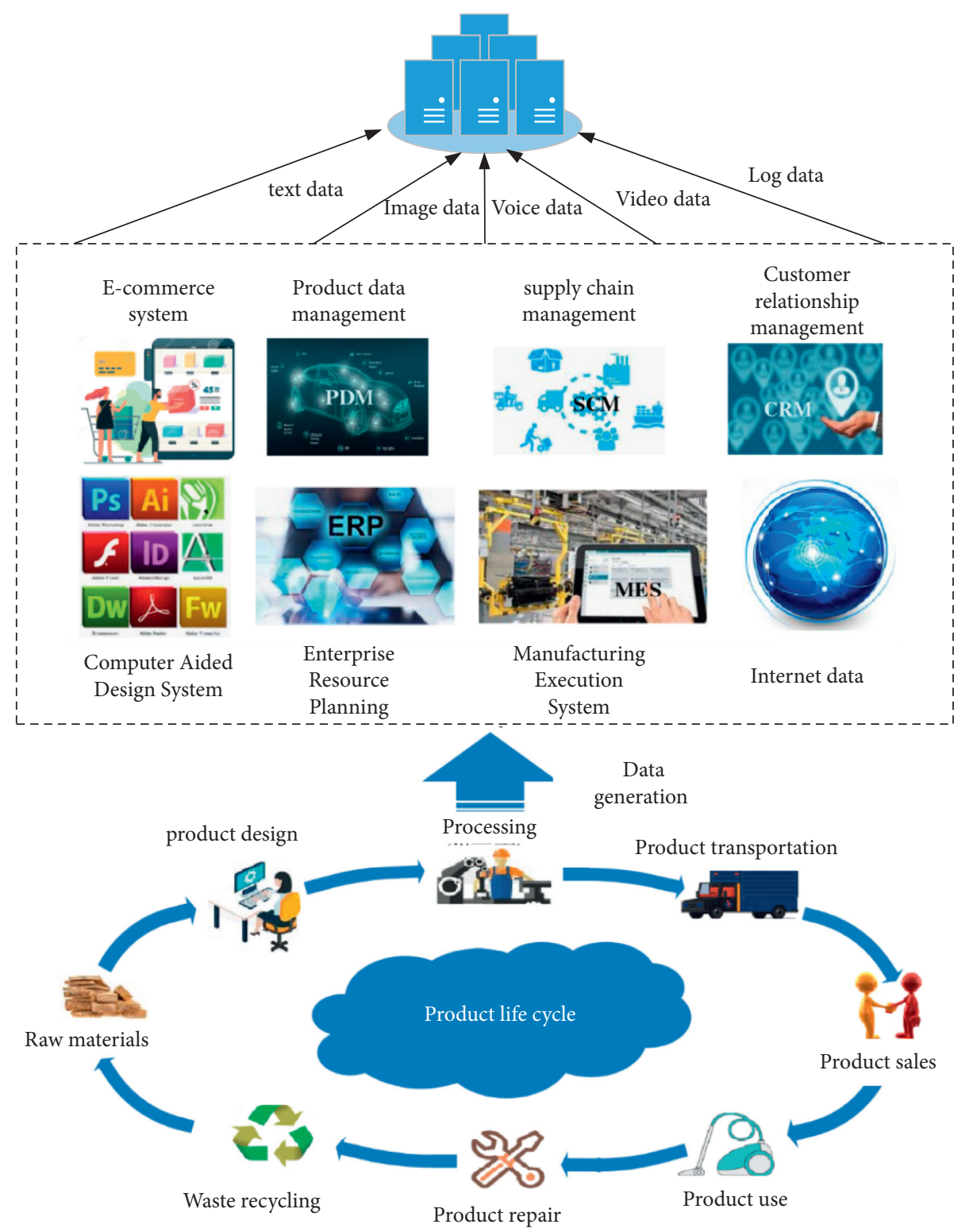

Figure 1: Product life cycle data.

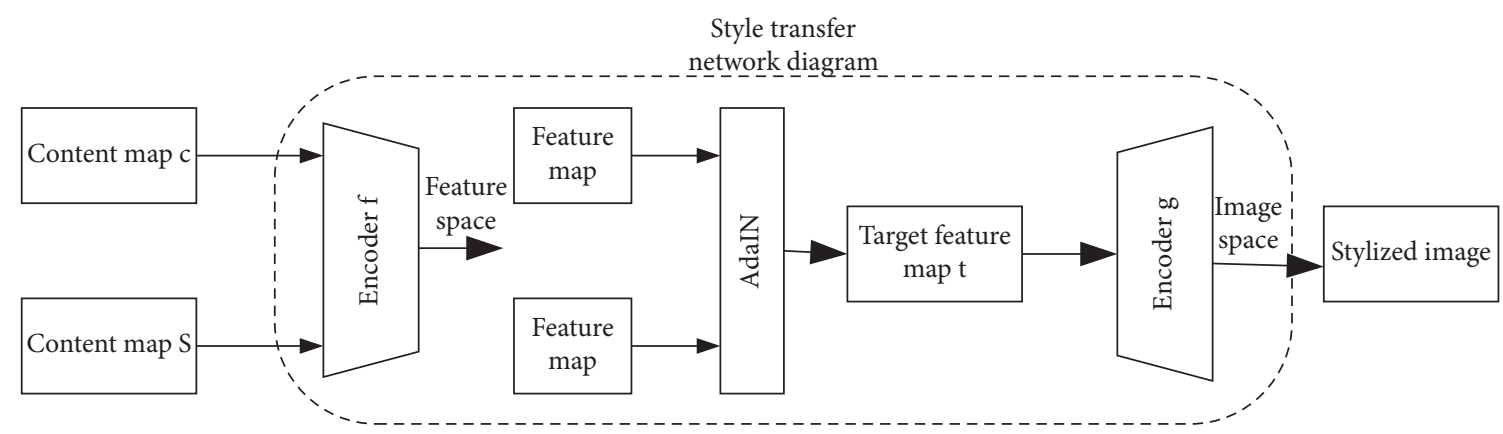

FIgURE 2: Neural style transfer. 


$$
L_{c}=f g(t)-t_{2} .
$$

Since the AdaIN layer transmits the mean and variance of the style features, the style loss can be obtained by matching the mean and variance:

$L_{s}=\sum_{i=1}^{K} \mu\left(\varnothing_{i} g(t)\right)-\mu\left(\varnothing_{i} s\right)_{2}+\sum_{i=1}^{K} \sigma\left(\varnothing_{i} g(t)\right)-\mu\left(\varnothing_{i} s\right)_{2}$,

where $\phi$ represents the pretrained VGG-19 model, $K$ represents the number of convolutional layers of the model, and $\phi_{i}(s)$ represents the activation value of the style map in the $i$ th layer of the model $\phi$.

3.3. Semantics of Product Packaging Design Style Diagram. Sensibility is the feeling that people produce after being stimulated by the outside world. Human sensibility has the attributes of vague and polysemous, including vision, hearing, touch, smell, and other aspects. It is a kind of recognition and comprehensive judgment of the object by the person himself. Perceptual engineering is a technology that combines perceptual and engineering. It aims to link people's emotional response with the design characteristics of products and attempts to transform emotions into measurable design elements. It is a user-centered design method. The process of perceptual engineering applied to product design is shown in Figure 3. The basic idea is to describe products from two different perspectives, product semantics, and product attributes and establish a mapping model between the two in the synthesis stage. Kansei engineering mainly includes four steps: product field selection, product semantic space establishment, product attribute space establishment, and mapping model construction [21].

Color perception is the psychological perception that light passes through the lens to stimulate the retina and is transmitted to the visual center of the brain through nerve fibers. It reflects the emotion and preference of people for color. Studies have shown that, in people's psychological reactions to objects, the influence of color in the first 5 minutes accounts for $50-80 \%$, and after 5 minutes, it remains unchanged at 50\%. It can be seen that color has a greater impact on human sensory characteristics. In the field of image color research, Ishihara et al. tried to use self-organizing neural networks to construct a perceptual engineering expert system for monochrome images. Hsiao applies fuzzy set theory to product color planning, but only considers the evaluation of monochromatic images. Tsai and Chou proposed a two-color emotional model based on gray theory. However, when the product contains multiple components of different colors, predicting the evaluation of the entire image is much more complicated. Chen Lili et al. used perceptual engineering methods to analyze the color and volume of different wavelengths ("reduced-enlarged"), distance ("highly cold-close"), soft and hard feeling ("soft-hard"), sense of lightness ("light-heavy"), sense of warmth ("passionate-high cold"), mood ("relaxed-depressive"), personality ("exaggerated-low-key"), and texture ("Gorgeous-Plain") The correlation of the seven color semantic dimensions, as well as the relationship between sensory characteristics and stimulus response time, and the results are shown in Table 1 . The results in Table 1 show that the long-wavelength colors give people a strong sense of intimacy, publicity, and gorgeousness, while the short-wavelength colors are high-cold, low-key, and simple. This section uses the above research conclusions to evaluate the semantics of product packaging design style diagrams. In the selection process of product packaging style images, images composed of colors with similar wavelengths are consciously selected as the style map [22].

\section{Construction of Aided Design System for Product Personalized Packaging Driven by Big Data}

4.1. Data Processing. Big data have rich data types, including voice data, video data, image data, text data, geographic location data, and numerical data. These data can be divided into three types: structured, semistructured, and unstructured data. Among them, structured data are composed of defined data types, usually stored in relational databases (RDBMS), and have the characteristics of easy retrieval; the structure and content of semistructured data are mixed storage, which makes it strong flexibility. Common semistructured data include webpages and reports; unstructured data have the characteristics of large volume, high growth rate, and high value and are difficult to retrieve. Common unstructured data include text, images, and audio. Compared with the first two types of data, unstructured data processing is more difficult, but it contains a high value [23].

The purpose of data preprocessing is to make the original data meet the minimum specifications and standards for data analysis. The data generated during the product packaging design life cycle contain a large amount of data, which will bring difficulties and deviations to data analysis and require preprocessing. Data preprocessing mainly deals with missing data, abnormal data, duplicate data, logical error data, and inconsistent data; data integration is to merge and store data obtained from multiple data sources, and it is necessary to resolve multiple entity name recognition, redundancy, and data conflicts. Data transformation is to normalize, discretize, and sparse data to make the data suitable for data analysis. Normalization is to solve the dimensional difference between different features; data specification is a way to maintain the integrity of the original data. The operation of reducing the data scale under the premise can improve the efficiency of data analysis.

Big data have the characteristics of low value density and high commercial value. How to dig out valuable information from large-scale data is the core part of the big data processing process. The process of using analytical techniques to reveal valuable rules and results from data is called data analysis. Commonly used big data analysis techniques include machine learning, deep learning, natural language processing, and statistical analysis. Many researchers have integrated big data analysis technology to build a data analysis work platform. As shown in Table 2, commonly used working platforms include WEKA, Mahout, RapidMiner, and KNIME. 


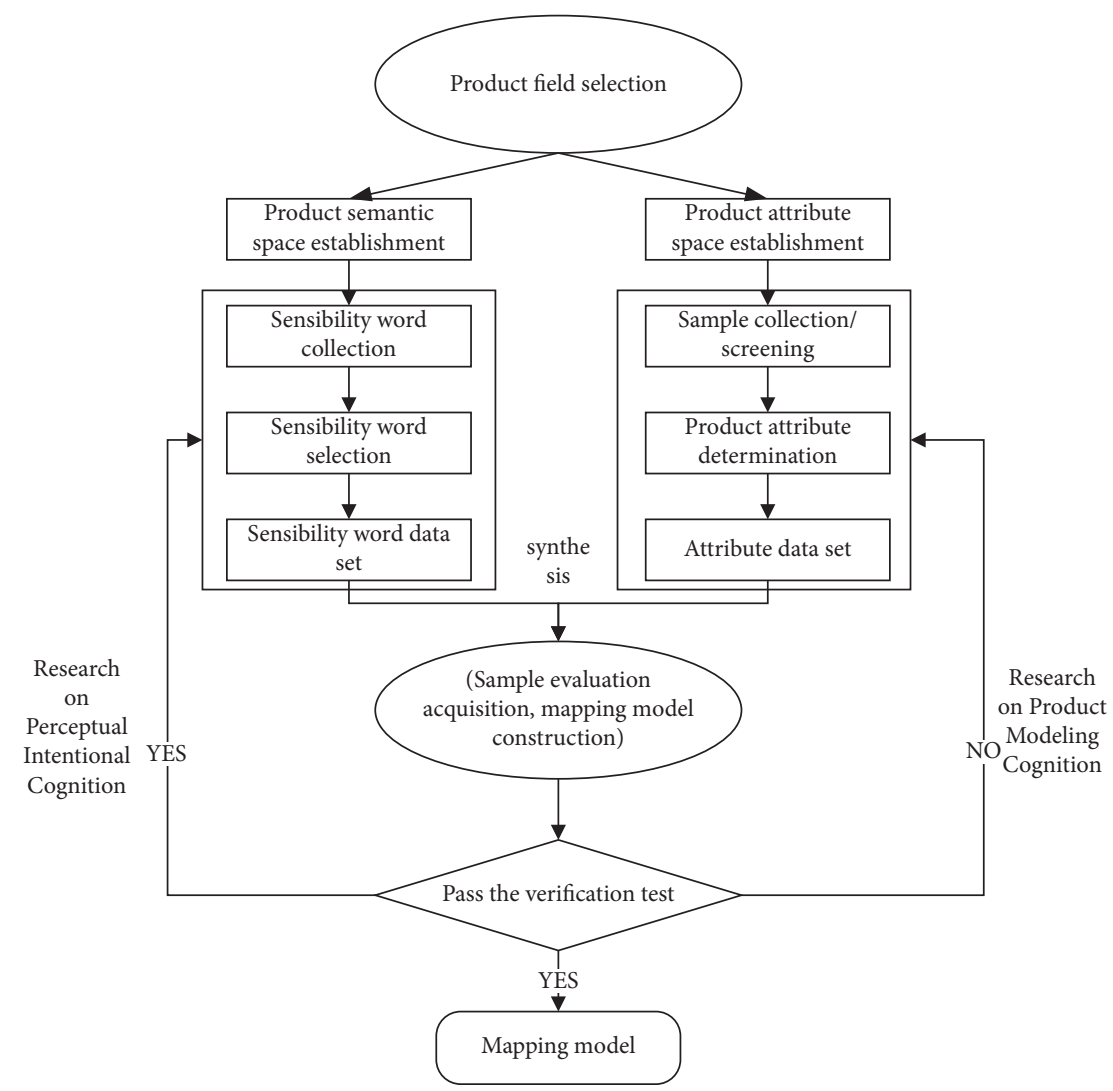

FIGURE 3: Kansei engineering process.

TABLE 1: Color perception grade.

\begin{tabular}{lcccccccc}
\hline & $\begin{array}{c}\text { Volume sense } \\
\text { reduced- } \\
\text { enlarged }\end{array}$ & $\begin{array}{c}\text { Sense of } \\
\text { distance cold- } \\
\text { close }\end{array}$ & $\begin{array}{c}\text { Soft and } \\
\text { hard soft- } \\
\text { stiff }\end{array}$ & $\begin{array}{c}\text { Soft and } \\
\text { hard soft- } \\
\text { stiff }\end{array}$ & $\begin{array}{c}\text { Soft and } \\
\text { hard soft- } \\
\text { stiff }\end{array}$ & $\begin{array}{c}\text { Mood relaxed- } \\
\text { depressive }\end{array}$ & $\begin{array}{c}\text { Character } \\
\text { public-low key }\end{array}$ & $\begin{array}{c}\text { Texture } \\
\text { gorgeous- } \\
\text { plain }\end{array}$ \\
\hline Red & 2.167 & 1.778 & 1.111 & -2.611 & 1.306 & 1.139 & -2.583 & -2.417 \\
Orange & 1.806 & 1.944 & -1.000 & -0.889 & -2.028 & -0.639 & -2.083 & -1.056 \\
Yellow & 2.028 & 2.000 & -1.361 & -1.500 & -1.500 & -0.194 & -2.194 & -1.000 \\
Green & -1.194 & -0.778 & 0.556 & 0.306 & 0.722 & -1.500 & 0.889 & 0.278 \\
Blue & 0.056 & -0.750 & -1.639 & -2.194 & 0.444 & -2.333 & 0.861 & 0.833 \\
Blue & -1.028 & -1.500 & -0.833 & -0.306 & 1.167 & -1.389 & -0.333 & -0.139 \\
Purple & -1.056 & -1.389 & 1.194 & 1.056 & 1.083 & 1.056 & -0.306 & -2.000 \\
Pink & 0.861 & 2.278 & -2.361 & -2.056 & -0.611 & -1.083 & -1.056 & -0.028 \\
White & 1.722 & 0.528 & -1.222 & -2.556 & 0.944 & -1.556 & 1.222 & 1.778 \\
Black & -2.139 & -2.333 & 2.778 & 2.889 & 2.278 & 2.611 & 2.833 & 2.472 \\
Gray & -1.306 & -1.417 & 0.472 & 0.861 & 2.028 & 1.056 & 2.194 & 1.806 \\
\hline
\end{tabular}

\subsection{System Design}

4.2.1. Architecture Design. (1) System architecture selection: typical distributed system architectures include client/server (Client/Server, C/S) and browser/server (Browser/Server, $\mathrm{B} / \mathrm{S})$, two types; the structure of the two is shown in Figure 4.

The biggest difference between the $\mathrm{C} / \mathrm{S}$ structure and the $\mathrm{B} / \mathrm{S}$ structure is whether the application logic layer is independent. The application logic layer contains the algorithms and calculation processes required by the system functions. The $\mathrm{C} / \mathrm{S}$ structure includes both the presentation layer and the application logic layer on the client side, while the $\mathrm{B} / \mathrm{S}$ structure adds a WEB server, which is independent of the application logic layer. In the $\mathrm{B} / \mathrm{S}$ structure, all users can share the WEB server, and the client is only responsible for receiving user requests and displaying the response results, which reduces the client's requirements for computer configuration [24].

The $\mathrm{C} / \mathrm{S}$ structure and the $\mathrm{B} / \mathrm{S}$ structure are also quite different on the client. The $\mathrm{C} / \mathrm{S}$ structure requires special software to be installed on the client, while the $\mathrm{B} / \mathrm{S}$ structure can be accessed using a standard browser. The differences in the client side make the $\mathrm{C} / \mathrm{S}$ structure more complicated in terms of system upgrades, installation, maintenance, and 
TABLE 2: Data analysis work platform.

\begin{tabular}{|c|c|c|}
\hline $\begin{array}{l}\text { Work } \\
\text { platform }\end{array}$ & Advantage & Shortcoming \\
\hline WEKA & (1) The code is open source and can be used for secondary development & $\begin{array}{l}\text { (1) The effect of high-dimensional data is } \\
\text { poor }\end{array}$ \\
\hline & (2) Good effect of low-dimensional data & (2) Limited algorithm library \\
\hline & (1) Can be integrated with other big data platforms & (1) No graphical operation mode \\
\hline Mahout & $\begin{array}{l}\text { (2) Based on Hadoop implementation, suitable for processing large amounts } \\
\text { of data }\end{array}$ & (2) Use interface development \\
\hline RapidMiner & $\begin{array}{l}\text { (1) Fast integration } \\
\text { (2) Support multiple data resource access methods }\end{array}$ & (1) Poor parallelism \\
\hline KNIME & $\begin{array}{l}\text { (1) Simple operation process and intuitive results } \\
\text { (2) Complete data analysis methods }\end{array}$ & $\begin{array}{l}\text { (1) The integration is difficult } \\
\text { (2) Poor support for statistical models }\end{array}$ \\
\hline
\end{tabular}

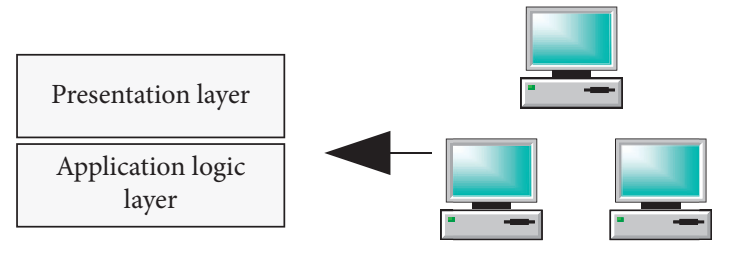

Client

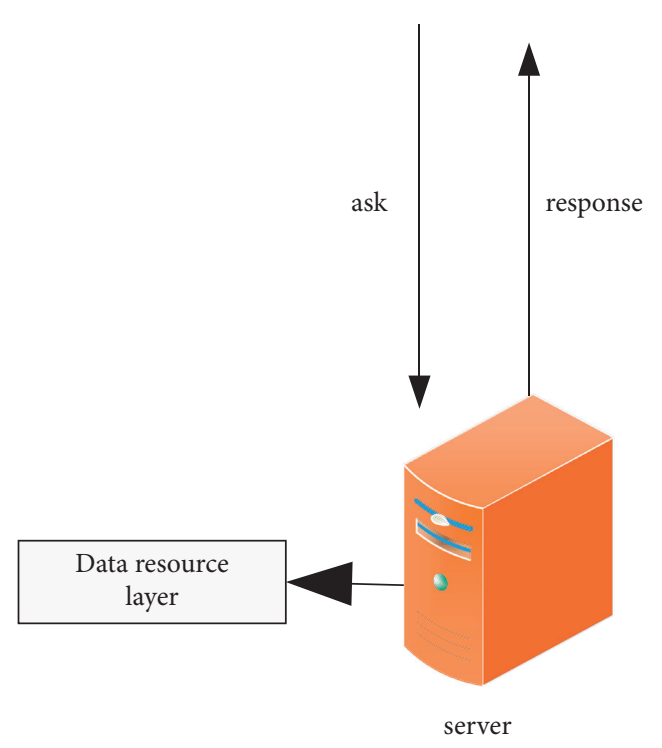

(a)

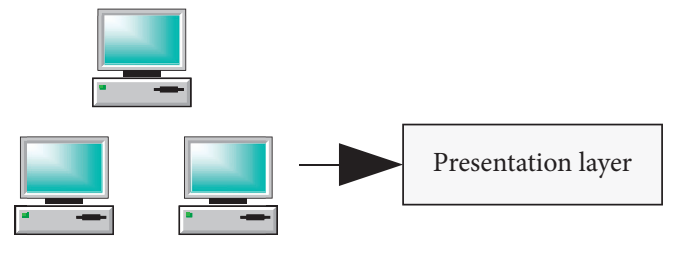

Client

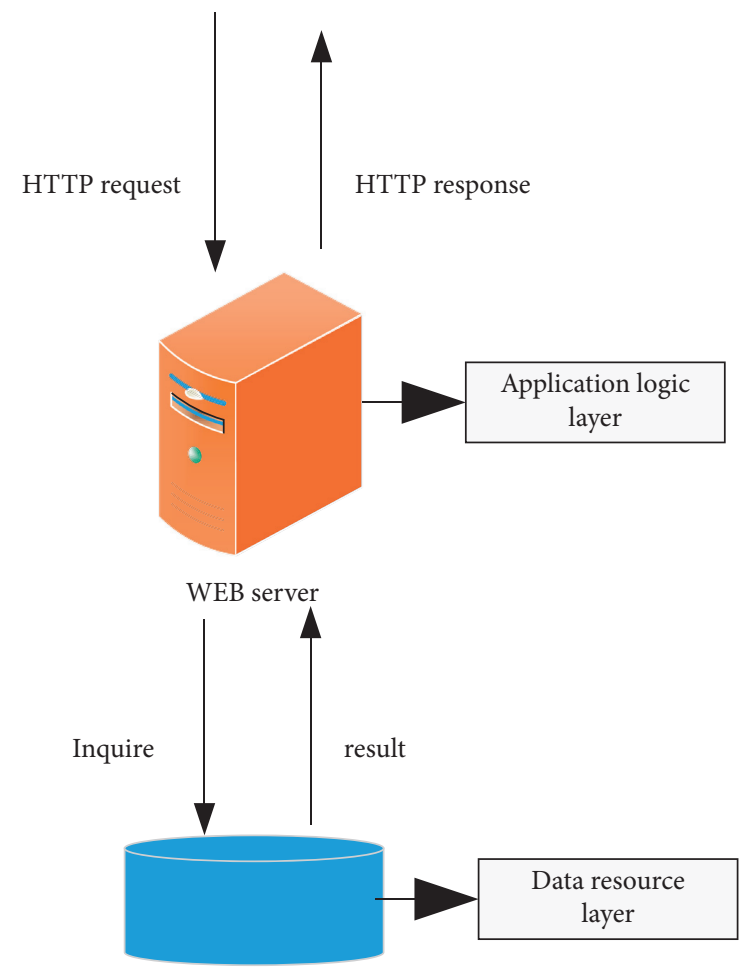

Database server

Figure $4: \mathrm{C} / \mathrm{S}$ and $\mathrm{B} / \mathrm{S}$ structure.

user training. Considering that the prototype system developed in this section faces a large number of users, and the functions of the system will be further enriched as the development progresses, a B/S structure with low technical requirements for users, low client computer configuration requirements, and easy update and maintenance is selected.
4.2.2. Database Design. (1) Choice of database: a database is a collection of data stored together in a certain way, which has the characteristics of low redundancy, easy expansion, high sharing, and strong independence. Users can add, delete, modify, and query data in the database. Databases can be divided into relational databases and nonrelational 


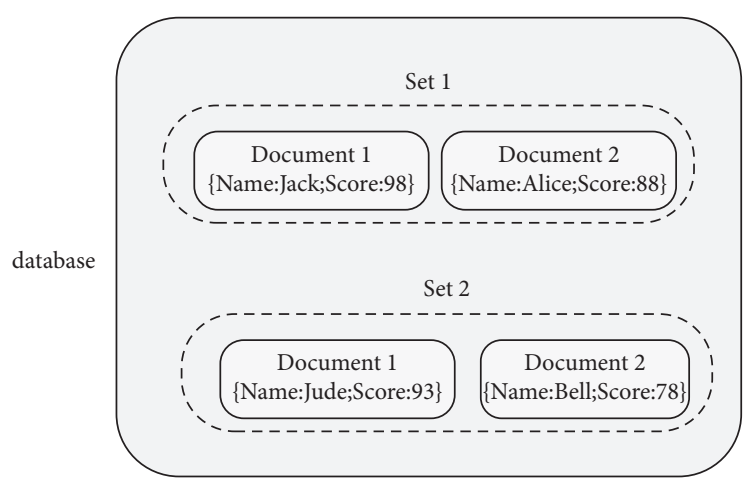

Figure 5: MongoDB database.

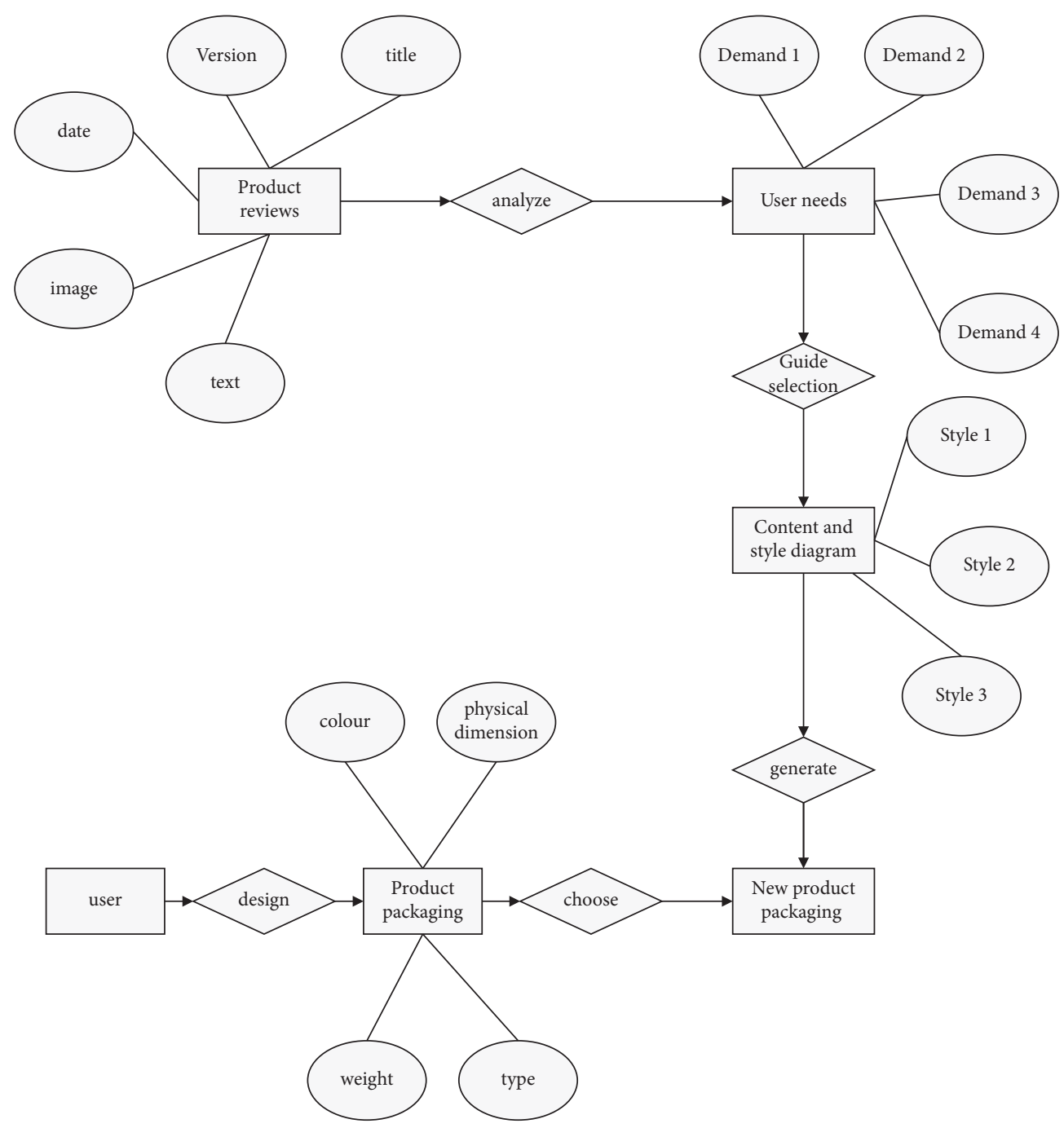

FIGURE 6: Big data-driven product innovation design auxiliary prototype system.

databases. Relational databases use a two-dimensional table model to organize data. They have the characteristics of consistent data format and easy management and operation. The mainstream relational databases include DB2, Microsoft SQL Server, Microsoft Access, and MySQL. Nonrelational database is an extension and extension of relational data. Its storage format is very flexible, including document format, key-value format, and image format. Due to the complex data types involved in the prototype system to be developed, this section uses a nonrelational database for storage. MongoDB is a commonly used nonrelational database with high performance, easy deployment, easy use, and scalability. As shown in Figure 5, MongoDB consists of three parts: document, collection, and database. Key-value pairs 
form a set of documents; multiple records composed of multiple sets of documents are called collections, and multiple collections form a database.

(2) Data structure design: according to the functional requirements of the big data-driven product innovation design auxiliary prototype system, the database has designed product online comment information (Comment_information), product attribute information (Attributes_information), content image information (Content_image), style image information (Style_image) and objective weight values,ilnformation (Objective_weight), and other collections. The entity-relation diagram (ER) of the prototype system database is shown in Figure 6.

Comment_information is used to store product comment information, including product category, product image, product version, comment date, and comment text. The MongoDB database has two methods for storing image data. The first is to convert the image into a binary format and store it as a dictionary key-value pair; the second is to use the MongoDB submodule GridFS for storage. This section uses the first method to store image data. The detailed field descriptions are shown in Table 3.

Style_image and Content_image are used to store style image and content image, respectively. The data structure of the two is the same, including image number and image, as well as the score value of the image in the system's predefined style type. The detailed fields are shown in Table 4.

Objective_weight is used to store the objective weight of the product. The subjective weight of the product is input by the user through the system interface, and the two together form a comprehensive weight value through the game theory method to rank the alternatives. Objective_weight includes the product category and the weight value of the product on several criteria predefined by the system. The detailed fields are shown in Table 5.

4.2.3. Development and Operating Environment. The overall operating framework of the entire big data-driven product innovation design auxiliary prototype system adopts the $\mathrm{B} / \mathrm{S}$ mode, uses the Python language to complete the development, and uses the MongoDB database to store data. The software configuration in the system development process is shown in Table 6.

4.2.4. System Implementation. (1) System login: users access the big data-driven product innovation design auxiliary prototype system through a standard browser. Tourist users need to register before they can log in and access the system. The login interface of the system is shown in Figure 7 . The registered user enters the system homepage interface, as shown in Figure 8, after logging in. In addition to the core functions of the system, system announcements and some application cases are also displayed on the system homepage. System announcements facilitate users to keep abreast of system update functions and notifications of projects that users participate in; the application case part can help users better understand system functions.
TABle 3: Comment_information data structure.

\begin{tabular}{lccc}
\hline Serial & Number data name & Data type & Data description \\
\hline 1 & Product_category & String & Product name \\
2 & ID_id & Number & Product ID \\
3 & Product_image & Binary date & Product image \\
4 & Title & String & Title \\
5 & Product_version & String & Product version \\
6 & Comment_data & Date & Comment date \\
7 & Comment_user & String & Comment user \\
8 & Comment_test & String & Comment text \\
\hline
\end{tabular}

TABLE 4: Style_image data structure.

\begin{tabular}{lccc}
\hline Serial & Number data name & Data type & Data description \\
\hline 1 & Image_number & Number & Image number \\
2 & Image & Binary date & Image \\
3 & Style1_score & Number & Style 1 point \\
4 & Style2_score & Number & Style 2 point \\
5 & Style3_score & Number & Style 3 point \\
6 & Style4_score & Number & Style 4 point \\
7 & Style5_score & Number & Style 5 point \\
8 & Style6_score & Number & Style 6 point \\
\hline
\end{tabular}

TABLE 5: Objective_Weight data structure.

\begin{tabular}{lccc}
\hline Serial & Number data name & Data type & Data description \\
\hline 1 & Product_category & String & Product category \\
2 & Criterion1_weight & Number & Criterion 1 weight value \\
3 & Criterion2_weight & Number & Criterion 2 weight value \\
4 & Criterion3_weight & Number & Criterion 3 weight value \\
5 & Criterion4_weight & Number & Criterion 4 weight value \\
6 & Criterion5_weight & Number & Criterion 5 weight value \\
7 & Criterion6_weight & Number & Criterion 6 weight value \\
\hline
\end{tabular}

TABLE 6: Development environment software configuration.

\begin{tabular}{lc}
\hline Name & Tool \\
\hline Operating system & Ubuntu 18.04 \\
Development & P $y$ charm professional version:2018.1 \\
environment & Python,XML \\
Development language & MongoDB \\
Database & Tomcat, nginx \\
WEB server & B/S \\
Access mode & \\
\hline
\end{tabular}

4.3. Analysis of Test Results. Through the construction of the personalized packaging system established in this section of this article and the trained style transfer model, the content and style features in the content graph and style graph can be extracted and integrated separately through the style transfer model. The image generation module of the new product includes three parts: content map selection, style map selection, and image generation. As shown in Figure 8, the "Content Map" and "Style Map" columns, respectively, display the content map and style map selected by the user. After clicking the "Generate" button, the generation interface will automatically generate a new product image. 


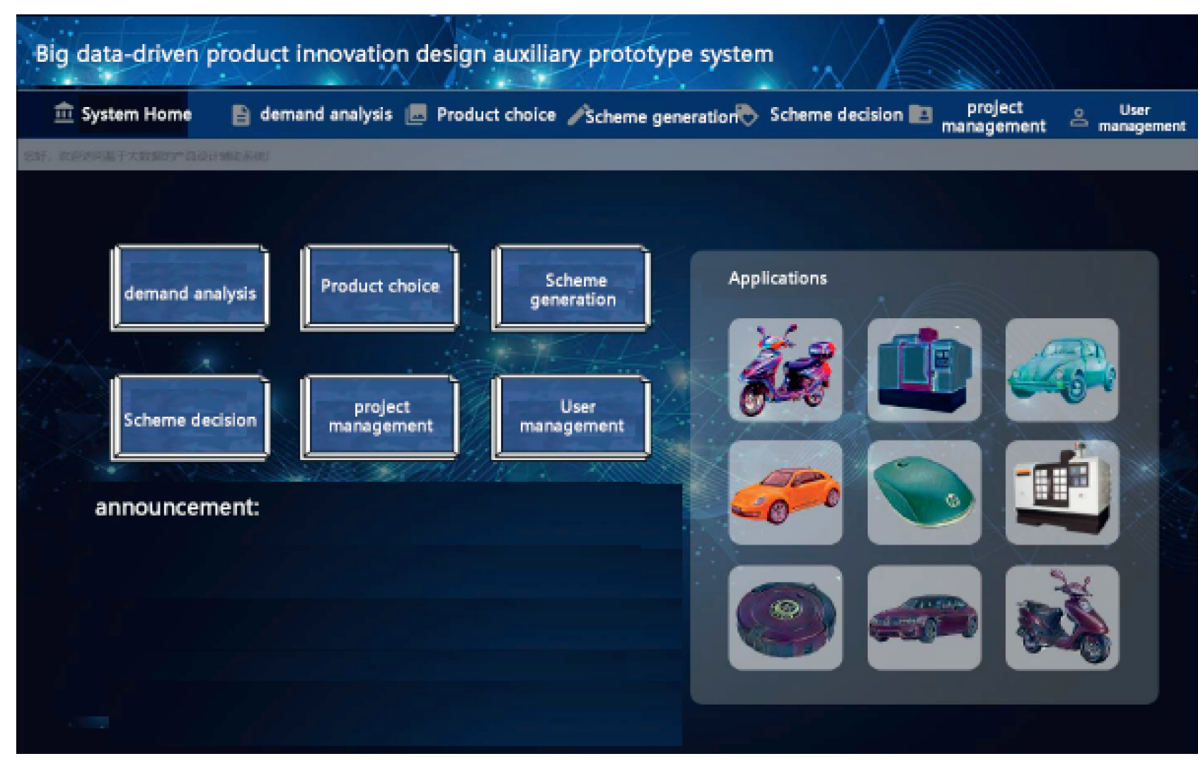

FIGURE 7: System login interface.

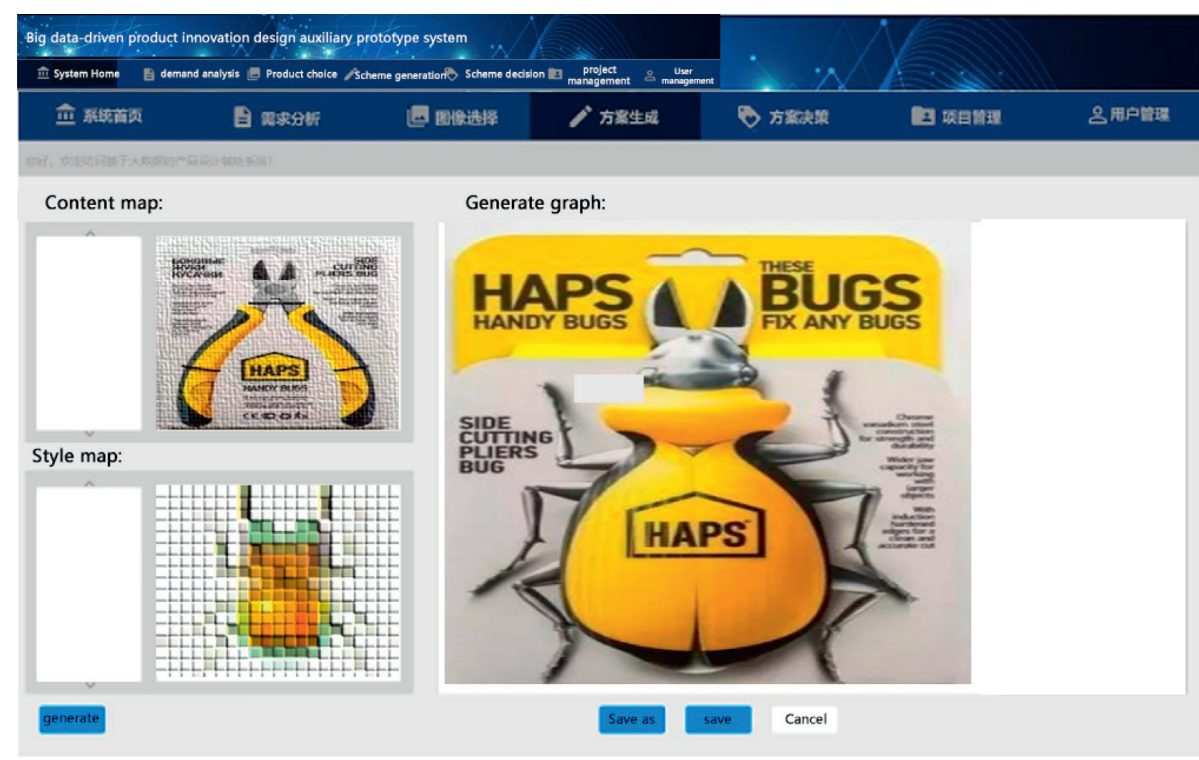

FIGURE 8: New product packaging design.

The generated new product image has both the product shape in the content map and the color characteristics of the style map. In addition, the plan generation interface also allows all users to upload local images for image generation, but the generated images cannot be stored in the system and can only be exported as jpg format and saved locally.

\section{Conclusion}

This study explores how to obtain information related to the product design from the big data generated in the product life cycle and uses machine learning, deep learning, and natural language processing technologies to conduct indepth analysis to further form intelligent and systematic products' innovative packaging design methods; the main research work is as follows. A set of big data-driven product innovation packaging design auxiliary prototype system is developed. First, it analyzes the design-related data generated in the product life cycle and the functional requirements of the system; secondly, the technical framework is designed according to the functional requirements; finally, the two core functions of the system, such as new product generation and product design plan decision-making, are shown in interface.

The innovative design of product packaging in the new era involves the intersection of multiple disciplines, including industrial design, computer, and psychology. This study introduces theories of big data, machine learning, deep learning, and natural language processing technology into the field of product design and proposes intelligence. The 
innovative design method of the product has been verified by experiments. The following aspects will be perfected and improved in the next research work. This study only studies two kinds of big data, image and text, and the behavior big data generated by operations such as clicking and browsing is also of great significance for obtaining user needs. In the follow-up research, we will explore the application of more different types of big data in product innovation design.

\section{Data Availability}

The data used to support the findings of the study are available from the corresponding upon request.

\section{Conflicts of Interest}

The authors declare that they have no conflicts of interest.

\section{References}

[1] C. R. Wilkinson and A. De Angeli, "Applying user centred and participatory design approaches to commercial product development," Design Studies, vol. 35, no. 6, pp. 614-631, 2014.

[2] A. Burnap, Y. Liu, Y. Pan, H. Lee, R. Gonzalez, and P. Y. Papalambros, "Estimating and exploring the product form design space using deep generative models," in Proceedings of the ASME 2016 International Design Engineering Technical Conferences and Computers and Information in Engineering Conference, vol. 1-13, Charlotte, NC, USA, August 2016.

[3] D. V. Horn, A. Olewnik, and K. Lewis, "Design analytics: capturing, understanding, and meeting customer needs using big data," in Proceedings of the Asme International Design Technical Conferences, pp. 863-875, Chicago, IL, USA, August 2012.

[4] M. Saifullah, McC. Rebecca, McC. Adam, and V. V. Quan, "Effect of drying techniques and operating conditions on the retention of color, phenolics, and antioxidant properties in dried lemon scented tea tree (Leptospermum petersonii) leaves," Journal of Food Processing and Preservation, vol. 45, no. 3, 2021.

[5] R. Ireland and A. Liu, "Application of data analytics for product design: s," CIRP Journal of Manufacturing Science and Technology, vol. 23, pp. 128-144, 2018.

[6] H. Ah-Reum, B. Nam, B. R. Kim et al., "Phytochemical composition and antioxidant activities of two different color Chrysanthemum flower teas," Molecules, vol. 24, no. 2, 2019.

[7] S. Vinodh and K. J. Manjunatheshwara, "Application of fuzzy QFD for environmentally conscious design of mobile phones," Green and Lean Management, Springers, Berlin, Germanny, pp. 149-160, 2017.

[8] K. Chakraborty, S. Mondal, and K. Mukherjee, "Analysis of product design characteristics for remanufacturing using Fuzzy AHP and Axiomatic Design," Journal of Engineering Design, vol. 28, no. 5, pp. 338-368, 2017.

[9] S. Tripathi, L. L. Henrekin, C. D. Read, and K. F. Welke, "Identification of critical to quality elements for intensive care rounds by kano analysis," Pediatric Quality And Safety, vol. 2, no. 4, 2017.

[10] R. M. Wong and O. O. Adesope, "Meta-analysis of emotional designs in multimedia learning: a replication and extension study," Educational Psychology Review, vol. 33, pp. 1-29, 2020.
[11] A. G. María, V. Pardo, R. Lucía, and M. N. Daniel, "Do products respond to user desires? A case study. Errors and successes in the design Process, under the umbrella of emotional design," Symmetry, vol. 12, no. 8, 2020.

[12] M. Antoine, A. Marie-France, and B. Éric, "Ordinary users, precursory users and experts in the anticipation of future needs: evaluation of their contribution in the elaboration of new needs in energy for housing," Applied Ergonomics, vol. 94, 2021.

[13] G. Büyüközkan and F. Göçer, “Application of a new combined intuitionistic fuzzy MCDM approach based on axiomatic design methodology for the supplier selection problem," Applied Soft Computing, vol. 52, pp. 1222-1238, 2017.

[14] D. Mourtzis, E. Vlachou, and N. Milas, "Industrial big data as a result of IoT adoption in manufacturing," Procedia Cirp, vol. 55, pp. 290-295, 2016.

[15] M. Kopel, "Analyzing music metadata on artist influence," in Proceedings of the Intelligent Information \& Database Systems: Asian Conference, Bali, Indonesia, March 2015.

[16] H. Kim, "Towards a sales assistant using a product knowledge graph," Web Semantics Science Services and Agents on the World Wide Web, vol. 46, pp. 14-19, 2017.

[17] J. Ahn, J.-M. Park, L. Won-Ho, and G.-Y. Noh, "Website interactivity and processing: menu customization and sense of agency are keys to better interaction design," International Journal of Human-Computer Studies, vol. 147, 2021.

[18] D. Mourtzis, M. Doukas, and C. Vandera, "An ensemble deep convolutional neural network model with improved DS evidence fusion for bearing fault diagnosis," Sensors, vol. 17, no. 8, p. 1729, 2017.

[19] R. Estrada and I. Ruiz, Big Data SMACK: A Guide to Apache Spark, Mesos, Akka, Cassandra, and Kafka, Apress, New York, NY, USA, 2020.

[20] A. Kangale, S. K. Kumar, M. A. Naeem, M. Williams, and M. K. Tiwari, "Mining consumer reviews to generate ratings of different product attributes while producing feature-based review-summary," International Journal of Systems Science, vol. 47, no. 13, pp. 3272-3286, 2016.

[21] Pew Research Center, "Online reviews," 2019.

[22] E. Ilbahar and S. Cebi, "Classification of design parameters for E-commerce websites: a novel fuzzy Kano approach," Telematics and Informatics, vol. 34, no. 8, pp. 1814-1825, 2017.

[23] J. Vieira, J. M. A. Osório, S. Mouta et al., "Kansei engineering as a tool for the design of in-vehicle rubber keypads," Applied Ergonomics, vol. 61, pp. 1-11, 2017.

[24] M. Nagamachi, "Successful points of kansei product development," in Proceedings of the KEER2018, Go Green with Emotion. 7th International Conference on Kansei Engineering \& Emotion Research, vol. 19-22, no. 146, pp. 177-187, Kuching, Malaysia, March 2018. 